\title{
Analysis of Effect of Bisphosphonate for Radionuclide Uptake in Bone Scintigraphy during Cancer Treatment
}

\author{
Fatima Arfan ${ }^{1 *}$, Narjis Batool ${ }^{2}$, Nosheen Arshad ${ }^{3}$, Warda Mehak $^{4}$, Syed Naqi Abbas ${ }^{5}$, Abid Ali $^{6}$ \\ ${ }^{14},{ }_{5}$ Medical Imaging Doctor, Department of Radiological Sciences and Medical Imaging, University of Lahore, Gujrat, Pakistan \\ ${ }^{2}, 3$ 'Lecturer, Department of Radiological Sciences and Medical Imaging, University of Lahore, Gujrat, Pakistan \\ ${ }^{6}$ Associate Professor, Department of Allied Health Sciences, University of Lahore, Gujrat, Pakistan
}

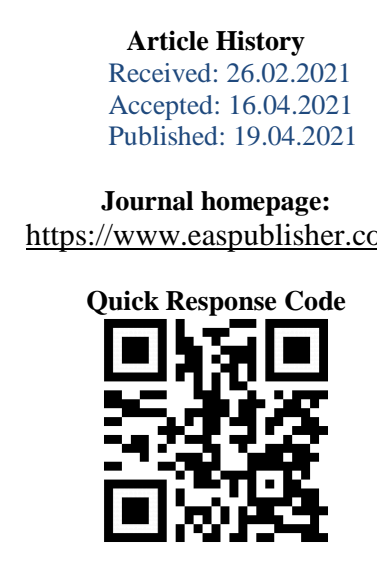

Abstract: Introduction: Bone scan is most commonly performed scan in nuclear medicine. There exists a relationship between bone scan and bisphosphonate therapy because after bisphosphonate therapy bone scan is mandatory. Optical scintigraphy and magnetic resonance imaging are two emerging molecular imaging methods used to test bone physiology. This review focuses on nuclear bone scintigraphy since it is the most well-established method with a large body of research data to back it up in clinical practice. The radionuclides technetium-99m (Tc-99m) and fluoride-18 are commonly used in bone nuclear scintigraphy (F-18). Tc-99m and F-18 are generally correlated with Medtronic destructive (Tc-99m MDP) and fluoride and sodium, respectively (F-18 NaF). The decay of photons from the radioisotope is captured by a nuclear camera holding a salt valuable stone after these particles are inserted intravenously. This is accomplished by the shimmering or fluorescence technique, which occurs when a photon emitted by a radionuclide collides with a salt useful stone within a nuclear camera. Methodology: It was an Observational study of 30 patients conducted at Department of Nuclear Medicine, INMOL Lahore from November 2020-February 2021. Cancer patients of both male and female genders receiving bisphosphonate therapies were included in this study. The age and gender matched patients were included. Objective: The aim of the research was to see how bisphosphonates affected oncology patients' bone scans. The key goal of this research was to see how bisphosphonates therapy affected bone scan. Results and Conclusion: The overall uptake of radiopharmaceutical in bone tends to increase after bisphosphonates therapy. The relationship between the bisphosphonate therapy given to the patients and their bone scan is significant. The study hypothesis is accepted that bisphosphonate. Hence effect of bisphosphonate therapy on skeletal system can be monitored by bone scan.

Keywords: Nuclear scintigraphy, bisphosphonate therapy, bone scan.

Copyright () 2021 The Author(s): This is an open-access article distributed under the terms of the Creative Commons Attribution 4.0 International License (CC BY-NC 4.0) which permits unrestricted use, distribution, and reproduction in any medium for non-commercial use provided the original author and source are credited.

\section{INTRODUCTION}

The number of oncology patients is quite large in Pakistan. They are usually given bisphosphonate therapy. Bone scan is most commonly performed scan in nuclear medicine. There exists a relationship between bone scan and bisphosphonate therapy because after bisphosphonate therapy bone scan is mandatory $[1,2]$. Optical scintigraphy and magnetic resonance imaging are two emerging molecular imaging methods used to test bone physiology [3]. The emphasis of this essay is on nuclear bone scintigraphy, since it is the most wellestablished method with a large body of research data to back up its usage in clinical medicine [4]. The radionuclides technetium-99m (Tc-99m) and fluoride18 are commonly used in bone nuclear scintigraphy (F18). Tc-99m and F-18 are generally correlated with Medtronic destructive (Tc-99m MDP) and fluoride and sodium, respectively (F-18 NaF). The decay of photons from the radioisotope is captured by a nuclear camera holding a salt valuable stone after these particles are inserted intravenously. This is accomplished using the shimmering or fluorescence method, which occurs when a photon emitted by a radionuclide collides with a salt useful stone within a nuclear camera. [4] Minerals 
$(65 \%)$, fibrillar organic matrix $(35 \%)$, cells, and water make up the composition of bone (nine).

The biological fluid contains mineralization inhibitors which we called inorganic pyrophosphate. Inorganic Pyrophosphate (PPi), has long been known as water softener due to inhibition of calcium carbonate as well as calcium phosphate crystal formation. Since hydrolytic enzymes in the gastrointestinal tract hydrolyze the P-O-P backbone, PPi is metabolically defective. So more attention in turned to stable analogs of PPi called bisphosphonates [7]. Bisphosphonates are a class of drugs that are analogue of inorganic pyrophosphates containing two C-P Bonds that inhabit bone resorption and calcification, built new bone by formation of osteoblast cells [8,9]. The retention of bisphosphonates through intestinal is low and variable (1-10\%). Uninvolved dissemination happens in the stomach and upper small digestive tract. On the off chance that bisphosphonates are given with calcium and iron its viability is diminished [10, 11]. 20-80\% of assimilated bisphosphonates quickly taken up bone and the update discharged in the pee, the half-existence of bisphosphonates in the course is short (0.5-2 hours). Statement of bisphosphonates happens at locales of bone resorption and development where there is osteoblast action. Because of this property of bisphosphonates, it is utilized in the atomic drug when it named with technetium 99 utilized for, bone filtering. At the point when bisphosphonates are taken up by bone and delivering an impact, bisphosphonates are put away in bone [12]. Bisphosphonates utilized clinically as skeletal markers as Tc-99m subsidiaries, patients with expanded bone obliteration as antiosteolytic operators, calcification inhibitors in patients with ectopic calcification and hardening. Bisphosphonates specialists were utilized for bone imaging, bone checking, despite everything they stay valuable for a location of bone injury and metastasis.

Bisphosphonates treatment decreased the frequency of myeloma, metastatic prostate disease, lung malignancy, strong tumors, and renal cell carcinoma $[13,14]$. The most ordinarily performed atomic medication check test is bone scintigraphy. Through Bone scintigraphy, the whole skeleton can be imaged. This test speaks to both morphology and physiology of bone while radiography just discloses to us morphology. For instance, the nearness of osteoblastic metastases from a breast tumor or prostate tumors can be seen. Sensitivity of the bone scintigraphy scan test is more than other imaging modalities $[15,16]$. The Explicitness of bone scintigraphy is restricted in this manner it is still suggested by consolidating with traditional radiographs or other imaging modalities, for example, CT or MRI [18].

\section{Materials ANd Methods}

From November 2020-February 2021, 30 patients participated in an observational analysis at the
Department of Nuclear Medicine, INMOL Lahore. This research involved both male and female cancer patients who were undergoing bisphosphonate therapy. Patients of similar ages and genders were included in the study. Individuals who failed to meet the above-mentioned criteria were automatically disqualified. All of the findings were interpreted using the Statistical Package for the Social Sciences (SPSS) version 20.0.

\section{Equipment}

All these cases were performed using: $99 \mathrm{Mo}$ $199 \mathrm{mTc}$ generator from which fresh technetium was eluted. Deluxe Isotope Calibrator II was used to measure the activity to be injected to the patient.Gamma camera with a wide field of view The collimator used was a low-energy all-purpose collimator with a $20 \%$ window based on $140 \mathrm{Kev}$. The patients were prior informed and prepared for the exam. The study was conducted after taking consent from the patients. Both qualitative and quantitative analysis was performed.

\section{Results}

It was an observational study conducted in the Department of Nuclear Medicine of INMOL Lahore including 30 patients. The data was collected using the conservative technique from November 2020-February 2021. The patients were split into two classes, each of 15 people. Group A was not given bisphosphonate therapy, whereas Group B was given bisphosphonate therapy. There was no distinction made between male and female participants; all were present. For each patient, a performer was created and filled out based on the patient's experience and imaging results. This research looked at the adult population. The average age is 55.63.97 years old. Patients in Groups A and B have a mean age of 53.939.72 and 57.339.85, respectively. The findings are shown in Table 4.1 .

The male to female ratios in groups $\mathrm{A}$ and $\mathrm{B}$ were $7(46 \%)$ and $8(54 \%)$ respectively, whereas the male to female ratios in groups B and C were $11(73 \%)$ and 4 respectively (27 percent) (Table 4.2). The patients studied in group A were with breast (11), prostate cancer (4) whereas the patients studied in group B were with breast (8) and prostate cancer (6) and lung cancer (1). (Table 4.3) Table 4.4 shows the frequency of Metastatic on bone scan in both the groups. The frequencies of Metastasis on bone scan in Group A was (+ve 4, -ve 11) while in Group B (+ve 13,-ve 2). To find the correlation a between bone scan and bisphosphonate therapy in cancer patients: the data were analyzed by applying $\mathrm{T}$ statistics and $\mathrm{P}$ value was determined. In group statistics, the mean for a bone to soft tissue ratio of a skull, pelvic bone and femur in group A and B were $(1.64 \pm 1.37),(2.08 \pm 2.26)$ and $(0.08 \pm 0.89)$ while in group. 
B $(3.55 \pm 1.94),(4.37 \pm 2.44)$ and $(1.88 \pm 1.31)$.

The $\mathrm{p}$-value for a bone to soft tissue ratio of a skull is
0.04 , the p-value for bone to pelvic bone ratio is 0.01 and bone to soft tissue ratio for femur bone is 0.01 .

Table-4.1: Mean age of Post-BT \& Pre BT groups $(n=30)$

\begin{tabular}{|l|l|}
\hline Groups & Means (Years) \\
\hline Group A(without bisphosphonate) & $53.93 \pm 9.72$ \\
\hline Group B( with bisphosphonate) & $57.33 \pm 9.85$ \\
\hline
\end{tabular}

Table-4.2 Gender distribution in bisphosphonate and without bisphosphonate therapy $(\mathrm{n}=30)$

\begin{tabular}{|l|l|l|l|}
\hline Gender & Bisphosphonate therapy & -Bisphosphonate therapy & Total \\
\hline Male & 7 & 4 & 11 \\
\hline Female & 8 & 11 & 19 \\
\hline Total & 15 & 15 & 30 \\
\hline
\end{tabular}

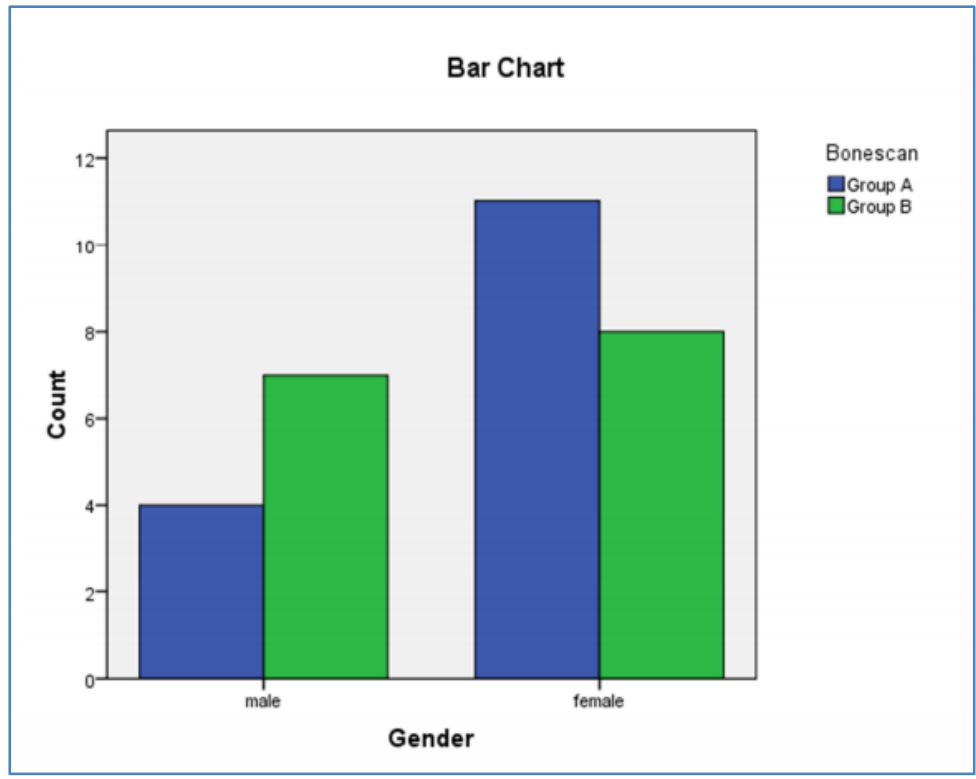

Fig-4.1

Table-4.3: Comparison of diagnosis among the patients with bisphosphonate andwithout bisphosphonate therapy $(\mathrm{n}=\mathbf{3 0})$

\begin{tabular}{|l|l|l|l|}
\hline Diagnosis & Pre-Bisphosphonate therapy & Post-Bisphosphonate therapy & Total \\
\hline Breast cancer & 11 & 8 & 19 \\
\hline Prostate cancer & 4 & 6 & 10 \\
\hline Lung cancer & 0 & 1 & 1 \\
\hline Total & 15 & 15 & 30 \\
\hline
\end{tabular}

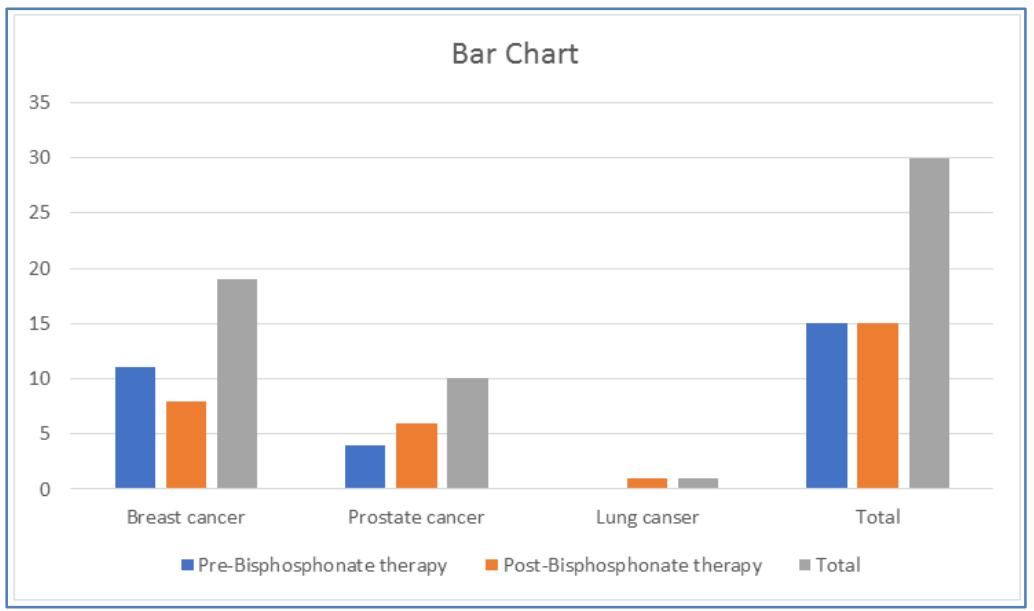

Fig-4.2: Graph of gender distribution in bisphosphonate and without bisphosphonate therapy $(\mathrm{n}=30)$ 


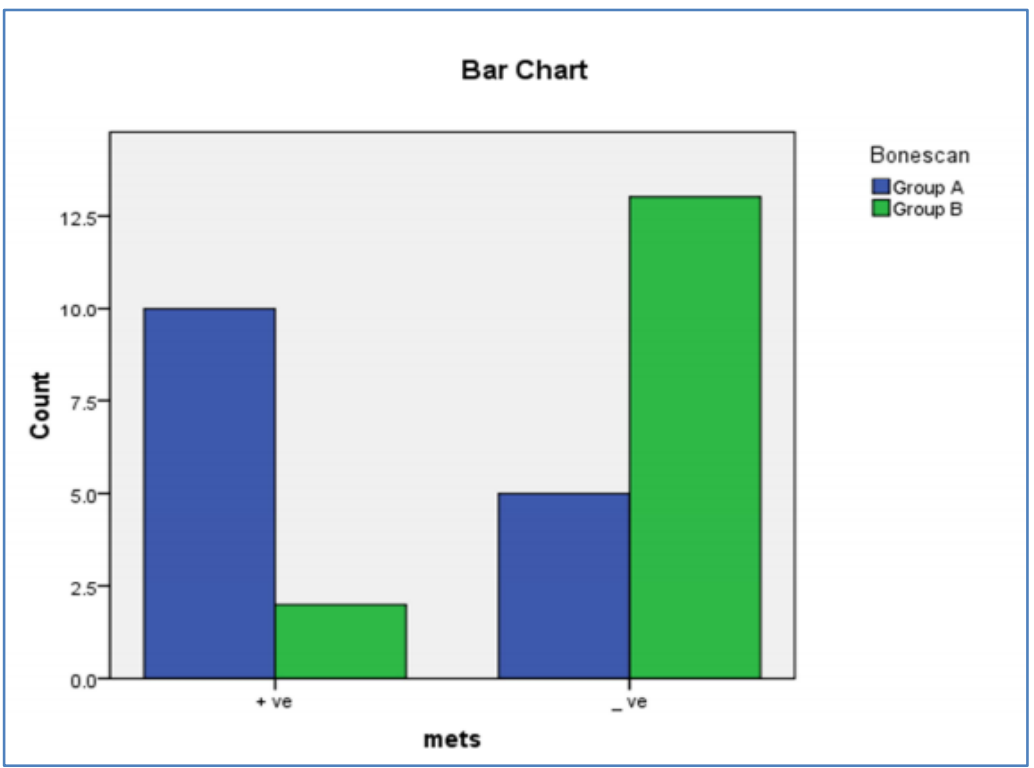

Fig-4.3: Graph of comparison of frequency of Mets in patients with bisphosphonate and without bisphosphonate therapy $(\mathbf{n}=\mathbf{3 0})$

Table-4.4: Comparison of Frequency of Mets in patients with bisphosphonate and without bisphosphonate therapy $(\mathbf{n}=\mathbf{3 0})$

\begin{tabular}{|l|l|l|l|}
\hline Metastasis & Pre-Bisphosphonate therapy & Post-Bisphosphonate therapy & Total \\
\hline$+\mathrm{ve}$ & 04 & 13 & 12 \\
\hline$-\mathrm{Ve}$ & 11 & 02 & 18 \\
\hline Total & 15 & 15 & 30 \\
\hline
\end{tabular}

Table-4.5: Comparison of bone to background ratio in patients with bisphosphonate and without bisphosphonate therapy $(n=30)$

\begin{tabular}{|l|l|l|l|l|}
\hline $\begin{array}{l}\text { Region of } \\
\text { Region of interest }\end{array}$ & & Mean & Std. Deviation & $\begin{array}{l}\text { P value (2- } \\
\text { tailed) }\end{array}$ \\
\hline Bone to soft \\
tissue ratio of & Without & 1.64 & 1.37 & \\
Skull & bisphosphonate & 1.64 & 1.37 & \\
\cline { 2 - 5 } & With & 3.55 & 1.93 & \multirow{2}{*}{$0.004^{*}$} \\
\hline bisphosphonate & 3.55 & 1.93 & \\
Bissue ratio of & Without & 2.08 & 2.28 & \multirow{2}{*}{$0.013^{*}$} \\
pelvic bone & bisphosphonate & 2.08 & 2.26 & \\
\cline { 2 - 5 } & With & 4.37 & 2.44 & \\
\hline Bone to soft & bisphosphonate & & 2.44 & \multirow{2}{*}{$0.017^{*}$} \\
tissue ratio of & Without & 0.848 & 0.89 & \\
Femur & bisphosphonate & 0.848 & 0.89 & 1.3 \\
\cline { 2 - 5 } & With & 1.88 & 1.3 & \\
\hline
\end{tabular}

\section{DiscuSSION}

The research involved 30 patients and was an observational study. The patients were split into two classes of 15 , each of 15 patients. Group A was not given bisphosphonate therapy, whereas Group B was given bisphosphonate therapy. There were no other supplements taken by the bisphosphonates patients.

The male to female ratio in groups A and B is $7(46)$ and 8(54), respectively, while the ratio in groups $\mathrm{C}$ and D is 11(73) and 4 respectively (27). Patients in Group A were on average 59.06 years old, and those in Group B were on average 56.33 years old. The patients studied in group A were with breast (11), prostate cancer (4) whereas the patients studied in group B were with breast (6), prostate cancer (8) and lung cancer (1). The frequencies of Metastasis on bone scan in both the groups were (+ve 13,-ve2) and (+ve 4,-ve 11). We had chosen the skull to soft tissue ratio because of thin skull bones. Hip bone has high cancellous part and marrow so pelvic bone to soft tissue ratio was selected. Femur to soft tissue ratio was taken as there is thick cortex in the femur. Bone to background tissue ratio was calculated in both groups. For skull and femur ROI has drawn for bone to background tissue ratio in the center of bone while for pelvic bone it has at the level of 
pelvic bone. The mean of a skull to background ratio was $(1.63 \pm 1.37)$ in Group A and (3.55 \pm 1.93$)$ in Group B. The mean of pelvic bone to background ratio was $(2.08 \pm 2.26)$ in Group A and $(4.37 \pm 2.44)$ in Group B. The mean of a femur to background ratio was $(0.84 \pm 089)$ in Group A and $(1.87 \pm 1.31)$ in Group B. A bone to soft tissue ratio of a brain, pelvic bone, and femur was calculated using $\mathrm{T}$ statistics. When patients who obtained bisphosphonates treatment were relative to those who did not, the $\mathrm{P}$ value was important.

It favored the increasing uptake of bone density and reduces the uptake in the soft tissue due to bisphosphonates in group A. Chavdaroal et al. [2] conducted the study on bone scintigraphy in the monitoring effect of bisphosphonates in bone metastasis. They found that treatment with zoledronic acid reduces the number of a bone lesion with at least 6 months therapy before a start. The number of a lesion in baseline bone scan showed therapeutic response in patients who received bisphosphonates therapy. So bone scintigraphy is a functional imaging modality for the assessment of the bisphosphonates. The results of study were similar to the previously conducted study discussed above. The study population was adult patients of oncology and it was on bisphosphonate therapy and other similarity of my study with the above studies was the use of bone scan to detect the uptake.

Morris et al. [11] conducted a study on effect of intravenous bisphosphonates on bone scan. As directed by the doctor, a baseline scan was done. Within 72 hours, patients received intravenous bisphosphonate with zoledronic acid. A second bone scan was performed 72 hours after beginning zoledronic acid. The cumulative amount of bone metastases did not improve as a result of the paired imaging. The findings of a bone scan are unaffected by intravenous bisphosphonates, indicating that the bone scan is not capable of detecting this impact. The reason behind that is bisphosphonate does not show the significant result on short duration. The duration of my study was 3 months with 2 to 3 average therapies while this study was performed 72 hours. The result of Morris's study is not in congruence with my result. The study's result suggests that as the effect of bisphosphonates in Group $\mathrm{B}$ are detected on bone scan due to its higher specificity than other imaging modality.

\section{Conclusion}

The overall uptake of radiopharmaceutical in bone tends to increase after bisphosphonates therapy. The relationship between the bisphosphonate therapy given to the patients and their bone scan is significant. The study hypothesis is accepted that bisphosphonate. Hence effect of bisphosphonate therapy on skeletal system can be monitored by bone scan.

\section{REFRENCES}

1. Baert, L. (2008). Encyclopedia of Imaging. New York. Springer Publishers.

2. Chavdaroval, L., Piperkova, E., Tsonevska, A., Timcheva, K. and Dimitrova, M. (2006). Bone scintigraphy in the monitoring of treatment effect of bisphosphonates in bone metastatic breast cancer. J BUON, 11(4):499-504.

3. Donohoe, J. (2003). Society of Nuclear Medicine. PubMed. [online] Available at $<$ http://interactive.snm.org/docs/pg_ch34_0403 > [Accessed 25 Jan2014].

4. Elgazzar. (2004). Orthopedic Nuclear Medicine. New York. Spring Publisher

5. Fleisch, H. (2000). Bisphosphonates in Bone Disease: From the laboratory to the patient. 4th ed., New York: Elsevier Publishers.

6. Fleisch, H. (2000). Bisphosphonates in bone diseases. California: Elsevier Academic Press.

7. Fleisch, H. (2007).Introduction to bisphosphonate. History and Functional. Orthopade, 36(2):1034,106-9

8. Heymann, D. (2010). Bisphosphonates and bone disease: past, present and future. Curr Pharm Des, 16(27): 2948-9

9. Macro. (1995). Intravenous aminohydroxypropylidene bisphosphonate does not modify $99 \mathrm{mTc}$-hydroxymethylene bisphosphonate bone scintigraphy. Revue de Rhumatisme., 62(2):99-104.

10. Martin, T., \& Grill, V. (2000). Bisphosphonatesmechanisms of action. Experimental and clinical pharmacology, 9(4): 130-2.

11. Morris, P.G. (2010). Intravenous bisphosphonate therapy does not acutely alter nuclear bone scan results. Clin Breast Cancer, 10(1) 33-9.

12. Murphy, K. J. Line, B. R. and Malfetano, J. (1997). Etidronate therapy decreases the sensitivity of bone scanning with methylene diphosphonate labeled with technetium-99m. Candian Association of Radiologist Journel, 48(3):199-202.

13. Patel, S. (1995). Quantitative bone scintigraphy in the management of monostatic Paget's disease of bone.Arthritis \& Rheumatism. Clinical \& expermental metastasis.,38(10):1506-1239

14. Price, A. P. (2011). Skeletal imaging effects of pamidronate therapy in osteosarcoma patients. Pediatr Radiol., 42(4):451-8.

15. Roudier, M. P. (2003). Bone histology at autopsy and matched bone scintigraphy findings in patients with hormone refractory prostate cancer: the effect of bisphosphonate therapy on bone scintigraphy results. Clinical \& Experimental Metastasis. 20(2):171-80.

16. Russel, G. and Graham, R. (2007). Bisphosphonate: Mode of Action and Pharmacolog.Pediatrics, 199(2):150-162

17. Weissma, B.N. (2009). Imaging of Arthritis and Metabolic Bone China: Elsevier Health Science. 
18. Widler, L., Jahnke, W, and Green, J. R. (2012). The chemistry of bisphosphonates: from antiscaling agents to clinical therapeutics. Anticancer Agents Med Chem, 12(2): 95-101.

19. Donohoe, K. J., Henkin, R. E., Royal, H. D., \& Brown, M. L. (1996). Procedure guideline for bone scintigraphy: 1.0. The Journal of Nuclear Medicine, 37(11), 1903.

20. Lou, J., Wen, G., Dong, K., \& Zhang, Y. (2017). Early monitoring of osteoporosis treatment response by technetium-99m-methylene diphosphonate bone scan. Nuclear medicine communications, 38(10), 854-857.

21. Prior, J. O., Barghouth, G., Delaloye, J. F., Leyvraz, S., \& Delaloye, A. B. (2003). The value of bone marrow scintigraphy using $99 \mathrm{mTc}$ monoclonal antigranulocyte antibodies in complement to bone scintigraphy in detecting bone metastases from primary breast cancer. Nuclear medicine communications, 24(1), 29-36.
22. Ryan, P. J., Gibson, T., \& Fogelman, I. (1992). Bone scintigraphy following intravenous pamidronate for Paget's disease of bone. Journal of nuclear medicine: official publication, Society of Nuclear Medicine, 33(9), 1589-1593.

23. Vellenga, C. J., Bijvoet, O. L., \& Pauwels, E. K. (1988). Bone scintigraphy and radiology in Paget's disease of bone: a review. American journal of physiologic imaging, 3(3), 154-168.

24. Carrasquillo, J. A., Whatley, M., Dyer, V., Figg, W. D., \& Dahut, W. (2001). Alendronate does not interfere with $99 \mathrm{mTc}$-methylene diphosphonate bone scanning. Journal of Nuclear Medicine, 42(9), 1359.

25. Pecherstorfer, M., Schilling, T., Janisch, S., Woloszczuk, W., Baumgartner, G., Ziegler, R., Ogris, E. (1993). Journal of Nuclear Medicine: Official Publication, Society of Nuclear Medicine, 34(7):1039-1044

Cite This Article: Fatima Arfan et al (2021). Analysis of Effect of Bisphosphonate for Radionuclide Uptake in Bone Scintigraphy during Cancer Treatment. EAS J Radiol Imaging Technol, 3(2), 73-78. 